\title{
A rare midbrain infarction presenting with plus- minus lid syndrome with ataxia: a case report
}

\author{
Khalid Alsherbini", Kevin Kapadia and Justin A Sattin
}

\begin{abstract}
Introduction: We present the case of a patient with midbrain infarction with an unusual clinical presentation, where clinical diagnosis and anatomical localization were valuable tools in deciding treatment.

Case presentation: Our patient was a 59-year-old, right-handed Caucasian man with hypertension who presented to our facility with acute diplopia that persisted until he developed complete right-sided ptosis. He also had difficulty walking and coordinating movements of his upper extremities bilaterally, but this was worse on his left side.

Conclusions: Plus-minus lid syndrome with ataxia is a rare presentation of midbrain infarction with a unique localization and anatomical description. This case highlights the importance of clinical skills for making a diagnosis in the absence of imaging to confirm the findings.
\end{abstract}

\section{Introduction}

A total of $20 \%$ of ischemic strokes involve structures supplied by the posterior (vertebrobasilar) circulation. Many cases remain undiagnosed or incorrectly diagnosed because of the atypical clinical presentation and the lower sensitivity of diagnostic imaging of the posterior fossa. Although lacunar infarctions are usually less than $15 \mathrm{~mm}$ in diameter, the symptoms might be clinically devastating, especially when the midbrain or pons is affected. Such lesions often are very challenging to localize and require a thorough knowledge of neuroanatomy.

\section{Case presentation}

Our patient was a 59-year-old, right-handed Caucasian man with hypertension who presented to our facility with acute diplopia that persisted until he developed complete right-sided ptosis. He also had difficulty walking and coordinating movements of his upper extremities bilaterally, but this was worse on his left side.

Physical examination showed a complete, pupil-sparing, right oculomotor nerve palsy (Figure 1 and Figure 2 ). He had left eyelid retraction that did not correct

\footnotetext{
* Correspondence: kalsherbini@uwhealth.org

Neurology Department, University of Wisconsin - Madison, 1685 Highland Avenue, Medical Foundation Centennial Building, Madison, WI 53705-2281, USA
}

(c) 2011 Alsherbini et al; licensee BioMed Central Ltd. This is an Open Access article distributed under the terms of the Creative Commons Attribution License (http://creativecommons.org/licenses/by/2.0), which permits unrestricted use, distribution, and reproduction in any medium, provided the original work is properly cited. upon passively elevating the right eyelid, indicating that the retraction was not due to increased levator palpebrae superioris activity from attempted fixation with the ptotic eye (Figure 2). There was impaired upward gaze in the left eye due to superior rectus weakness (Figure 3 ) with intact medial rectus (Figure 4). He had no fatigable weakness and no sign of inflammation of the eye or surrounding soft tissues; a magnetic resonance imaging (MRI) scan of his brain showed no restricted diffusion.

On follow-up six months after an acute rehabilitation stay, his ptosis had resolved but he continued to have diplopia due to residual oculomotor paresis. His ataxia had improved with physical therapy such that he was able to walk with a cane inside his house and with a walker for longer distances.

\section{Discussion}

The finding of ptosis on one side and eyelid retraction on the other is known as plus-minus lid syndrome, and was originally described by Gaymard et al. [1]. It is usually caused by injury to the third nerve fascicle and the nucleus of the posterior commissure, although it has also been described in ocular myasthenia gravis [2].

Two muscles control the eyelid: one is the superior tarsal muscle (Müller's muscle), which is innervated by sympathetic fibers and is thus affected in Horner's syndrome. The other is the levator palpebrae superioris, a
Ciomed Central 


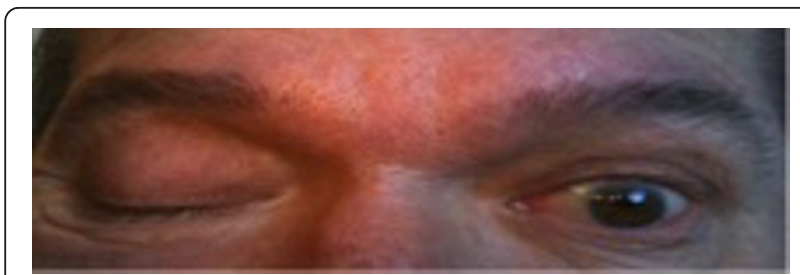

Figure 1 Our patient's right eye showing complete ptosis with left lid retraction.

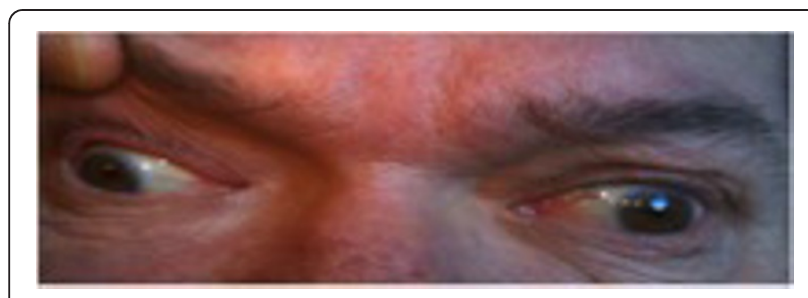

Figure 2 Our patient's right eye deviated down and out with no correction of the left eye retraction with elevation of the right eyelid.

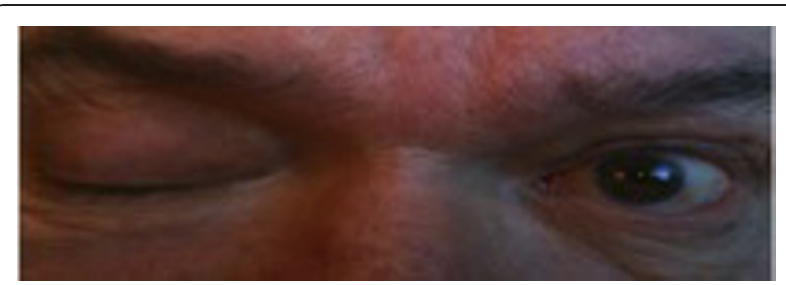

Figure 3 Impairment of left eye upgaze due to superior rectus weakness; our patient was asked to look up and to the left.

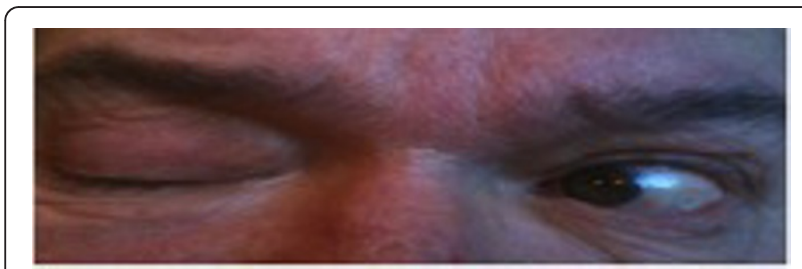

Figure 4 Intact medial rectus muscle on the left

skeletal muscle innervated by oculomotor fibers originating from the central caudal subnucleus of the third nerve complex $[3,4]$.

The nucleus of the posterior commissure sends inhibitory fibers to the central caudal nucleus. Lesions of this structure result in disinhibition of levator palpebrae superioris bilaterally. When the oculomotor fascicle is also involved, as in our patient's case, the ipsilateral eyelid retraction is masked by the ptosis resulting from the infra-nuclear third nerve palsy [2-4].
The contralateral ataxia was attributed to involvement of the superior cerebellar peduncle (Claude syndrome). The contralateral superior rectus weakness improved quickly, but serves as a reminder of the unique contralateral innervation of this muscle $[3,4]$.

An MRI of the brain had shown no restricted diffusion, which emphasizes the importance of making a clinical diagnosis. In one study, $25 \%$ of clinically diagnosed patients who had had a stroke had a negative initial diffusion-weighted MRI scan, with $23 \%$ of those having evidence of infarction on a repeat MRI scan. Most such lesions were lacunar and in the brainstem [5].

\section{Conclusions}

Plus-minus lid syndrome with ataxia is a rare presentation of midbrain infarction with a unique localization and anatomical description. This particular case highlights the importance of clinical skills for making a diagnosis in the absence of imaging to confirm the findings.

\section{Consent}

Written informed consent was obtained from the patient for publication of this case report and any accompanying images. A copy of the written consent is available for review by the Editor-in-Chief of this journal.

\section{Authors' contributions}

KA analyzed and interpreted the initial data regarding our patient's presentation and performed the literature review, and wrote the initial manuscript. JS reviewed the initial draft and made corrections and final editing changes, and he added some neuro-anatomy review material. KK helped with the final editing and review of the manuscript, with finalizing of the images used. All three authors were the stroke care team for our patient. All authors read and approved the final manuscript.

\section{Competing interests}

The authors declare that they have no competing interests.

Received: 20 July 2011 Accepted: 25 October 2011

Published: 25 October 2011

\section{References}

1. Gaymard B, Lafitte C, Gelot A, de Toffol B: Plus minus lid syndrome. J Neurol Neurosurg Psychiatry 1992, 55:846-848.

2. Porta-Etessam J, Benito-Leon J, Berbel A, Martinez A: Plus minus lid syndrome without ophthalmoplegia. Eur J Neurol 1999, 6:107.

3. Kotwal VS, Shenoy W, Joshi SR, Aklujkar AP, Nadkarni NA, Shetty K, Benny R, Butala N, Patwardhan M: Plus minus lid syndrome with ataxia. J Assoc Physicians India 2005, 53:908-909.

4. Galetta SL, Gray LG, Raps EC, Grossman Rl, Schatz NJ: Unilateral ptosis and contralateral eyelid retraction from a thalamic-midbrain infarction. Magnetic resonance imaging correlation. J Clin Neuroophthalmol 1993, 13:221-224.

5. Sylaja PN, Coutts SB, Krol A, Hill MD, Demchuk AM, VISION Study Group: When to expect negative diffusion-weighted images in stroke and transient ischemic attack. Stroke 2008, 39:1898-1900.

doi:10.1186/1752-1947-5-525

Cite this article as: Alsherbini et al:: A rare midbrain infarction presenting with plus-minus lid syndrome with ataxia: a case report. Journal of Medical Case Reports 2011 5:525 